\title{
Proposta para auxílio no tratamento de ocorrências de segurança pública por meio da plataforma ServiceNow ${ }^{1}$
}

\section{Proposal for assistance in the treatment of public security occurrences by means of the ServiceNow platform}

\author{
Almeida, Kenner Paulo de Morais ${ }^{1}$; Pina Filho, Armando Carlos de 2 \\ 1 Universidade Federal do Rio de Janeiro, Programa de Engenharia Urbana, \\ Escola Politécnica, Av. Athos da Silveira Ramos, 149, Cidade Universitária, \\ 21941-485, Rio de Janeiro - RJ, Brasil, kenneralmeida@poli.ufrj.br \\ 2 Universidade Federal do Rio de Janeiro, Programa de Engenharia Urbana, \\ armando@poli.ufrj.br
}

\begin{abstract}
RESUMO
A criminalidade está crescendo em todo o mundo, com ênfase na América Latina, onde as taxas são o dobro da média mundial, estando Brasil, México e Colômbia entre os países mais violentos. A tecnologia da informação pode ser uma importante aliada na resolução desse problema. Logo, o objetivo deste trabalho é demonstrar o potencial da plataforma ServiceNow para tratamento de ocorrências de segurança pública, considerando o registro da ocorrência realizado pelo cidadão, o direcionamento feito pelo Analista da Central de Atendimento para o batalhão policial adequado, o atendimento e a resolução da ocorrência, feitos pelo agente policial. No desenvolvimento deste trabalho foram utilizados variados recursos pré-existentes da Plataforma, customizados para ficarem aderentes ao processo de monitoramento de segurança pública. Foram implementadas configurações adicionais usando a linguagem de programação Javascript, buscando a criação do sistema a partir da seguinte metodologia: definir a aplicação desejada; fornecer os dados estruturais necessários; modelar os fluxos de trabalho para automatizar os processos de serviço; conectar aplicativos, dados e pessoas; e analisar as demandas em tempo real. Os recursos, praticidade e agilidade fazem com que a Plataforma seja uma interessante ferramenta para otimização e automação de processos de monitoramento aplicados em segurança pública.
\end{abstract}

Palavras-chave: segurança pública, plataforma ServiceNow, monitoramento.

\begin{abstract}
Crime is growing all over the world, with an emphasis on Latin America, where rates are twice the world average, with Brazil, Mexico and Colombia being some of the most violent countries. Information Technology can be an important ally in solving this problem. Then, the objective of this work is to demonstrate the potential of the ServiceNow platform for the treatment of tickets in public security, considering the registration of the occurrence carried out by the citizen, the appointment made by the Call Center analyst for the appropriate police department,

\footnotetext{
${ }^{1}$ ALMEIDA, Kenner Paulo de Morais; PINA FILHO, Armando Carlos de. Proposta para auxílio no tratamento de ocorrências de segurança pública por meio da plataforma ServiceNow. In: II SIMPÓSIO NACIONAL DE GESTÃO E ENGENHARIA URBANA: SINGEURB, 2019, São Paulo. Anais... Porto Alegre: ANTAC, 2019.
} 
attendance and resolution of the ticket, made by the police officer. For the development of this work, a variety of pre-existing features were used in the platform, customized to comply with the public security monitoring process. Additional configurations were implemented using the Javascript programming language, searching the creation of the system from the following methodology: define the desired application; provide the necessary structural data; model workflows to automate service processes; connect applications, data and people; and analyze the demands in real time. The features, practicality and agility make the Platform an interesting tool for optimization and automation of monitoring processes applied in public security.

Keywords: public security, ServiceNow platform, monitoring.

\section{INTRODUÇÃO}

O crescimento da criminalidade é um dos grandes problemas em todo mundo, como mencionado por Azevedo (2003), sendo que na América Latina o problema é ainda mais grave, já que as taxas de criminalidade são duas vezes maiores que a média mundial. Países como Brasil, México e Colômbia são os mais violentos. Diante deste cenário, governos e iniciativas privadas buscam soluções para resolver ou, ao menos, amenizar problemas da segurança pública (LIMA et al., 2016). Neste contexto, a Tecnologia da Informação surge como uma aliada, fornecendo uma gama de possibilidades que podem otimizar processos, auxiliar a tomada de decisões e resolução de problemas, por meio de recursos como inteligência artificial, aprendizado de máquina, geoprocessamento, dados em tempo real, redes sociais e tecnologias inclusivas, que contam com o apoio da sociedade para reportar problemas (PACHECO, 2018).

Máximo (2004) discorre que, embora as tecnologias estejam amplamente disponíveis em todo o mundo, há necessidade de inovar a prestação de serviços para torná-la mais ágil, precisa e com maior qualidade. Para isso é fundamental que os serviços públicos atualizem seus modos de trabalho, com investimentos em tecnologias e treinamento de pessoal. Além disso, a informatização dos serviços da Polícia Civil e da Polícia Militar é necessária, pois há uma infinidade de recursos computadorizados disponíveis, que podem ser extremamente proveitosos na elaboração de estratégias de monitoramento, controle, combate e prevenção ao aumento da criminalidade.

A Plataforma ServiceNow foi desenvolvida para automação de processos de negócios, operando em nuvem. Nela há módulos customizáveis de registro, acompanhamento e análise de ocorrências, e tem a vantagem de ser acessíveis em diferentes interfaces, como por computador ou por aplicativos de celular. Este trabalho almeja demonstrar o potencial da utilização da plataforma ServiceNow como ferramenta para o monitoramento de ocorrências de segurança pública, desde o registro da ocorrência por um cidadão até a resolução da mesma por um agente policial.

Dessa forma, para adaptação da plataforma foram utilizados diversos recursos pré-existentes, customizados para atender ao monitoramento de segurança pública, a saber: o aplicativo de celular, o processo nativo de Gestão de Serviço de Campo, as notificações de aplicativo (push notifications), relatórios e painéis. Foram implementadas configurações adicionais usando a linguagem de programação Javascript, buscando a criação do sistema a partir da seguinte metodologia: definir a aplicação desejada; fornecer os dados estruturais necessários; modelar os fluxos de trabalho para automatizar os processos de serviço; conectar aplicativos, dados e pessoas; e analisar as demandas em tempo real.

\section{SEGURANÇA PÚBLICA}

Furtado (2002) menciona que, em linhas gerais, o contexto da segurança está ligado à prática delituosa e a transgressão da lei, chamada de crime, sendo responsabilidade dos atores que representam a segurança pública, a redução dessas ocorrências. Entretanto, Azevedo (2003) também relata aumento contínuo da arbitrariedade, do abuso da força e da corrupção policial, bem como a sensação de insegurança e de temor ao crime entre a população civil. 
Segundo Andrade (2013), a criação de uma Conferência Nacional de Segurança Pública (CONSEG), protagonizada pelo Ministério da Justiça do Governo brasileiro, entre 2008 e 2009 , partiu do reconhecimento de que a segurança pública no Brasil envolve uma problemática de grande envergadura, que necessita evoluir em direção a um novo paradigma superador do paradigma punitivo que historicamente a orienta, a partir da definição constitucional da segurança como direito humano (IPEA, 2009).

A redução da criminalidade pode ocorrer por meio de duas formas: impedir que um crime aconteça e, caso ele tenha ocorrido, dissuadir sua repetição através da identificação, apreensão e punição dos culpados. Reuland (1997) indica que, diante desse grave cenário da segurança pública, a utilização intensiva de tecnologias de informação tem promovido uma verdadeira revolução no desempenho operacional das polícias ao redor do mundo.

Máximo (2004) relata que é difícil estimar o ritmo da mudança e o impacto de longo prazo das transformações tecnológicas aplicadas no policiamento, inclusive no mapeamento do crime, possibilitando melhor monitoramento, planejamento e respostas. Embora esses avanços prometem fornecer apoio ao controle de crimes, convém citar que essas tecnologias são ferramentas e, como tais, produzem benefícios à sociedade dependendo dos agentes humanos que as controlam, sendo necessárias constantes atualizações para garantir melhor agilidade, precisão e qualidade. Complementando, Azevedo (2006) relata que o policiamento é mais eficaz quando há apoio efetivo da população no monitoramento da criminalidade.

Dessa forma, o desenvolvimento e a aplicação de tecnologias que possibilitem o engajamento da sociedade podem ser aliados essenciais no reporte, no monitoramento e na resolução da incidência da criminalidade, contribuindo para uma significante melhoria da segurança pública (CARVALHO e SILVA, 2011). O aporte desses dados pode ser realizado através da utilização da plataforma ServiceNow como ferramenta para processo de segurança pública, contemplando o registro, o roteamento, o monitoramento e a resolução de uma ocorrência.

\section{A PLATAFORMA SERVICENOW}

A ServiceNow é uma empresa fundada em 2004 por Fred Luddy, atuando no segmento de computação em nuvem por meio de uma plataforma única para automação de processos de negócio (SERVICENOW, 2018), na qual empresas e instituições podem automatizar, de maneira inteligente, tarefas e fluxos de trabalho que integram sistemas, pessoas e dados. Algumas das principais vantagens da plataforma são: a rápida criação de soluções personalizadas, o uso de chatbots com tecnologia de inteligência artificial, aprendizado de máquina para dar mais eficiência aos processos, experiência de usuário mais intuitiva e moderna e suporte a aplicativos para dispositivos móveis.

Segundo publicado por Gartner (2018), a plataforma ServiceNow engloba todos os serviços em um único sistema, com ênfase em fluxos de trabalho e integrações, além de suportar variadas aplicações. A ServiceNow tem investido fortemente em recursos avançados, sendo amplamente conhecida por sua solução Saas (Software as a Service) de gerenciamento de serviços de $\mathrm{Tl}$, que foi desenvolvida como um exemplo para se apresentar os recursos da plataforma. A Figura 1 exibe as categorias de produtos da plataforma ServiceNow.

\subsection{Aplicação da plataforma ServiceNow em segurança pública}

A avaliação da utilização da plataforma para monitoramento de dados sobre segurança pública considera o registro da ocorrência de segurança pública, o roteamento para o perfil solucionador e a resolução da mesma, como exibido no Quadro 1, onde constam casos de uso, atores, objetivos e canais utilizados. Convém recordar que foi criado na plataforma ServiceNow, de forma personalizada, um ambiente para registro dessas ocorrências. 
Figura 1 - Categorias de produtos da plataforma ServiceNow.

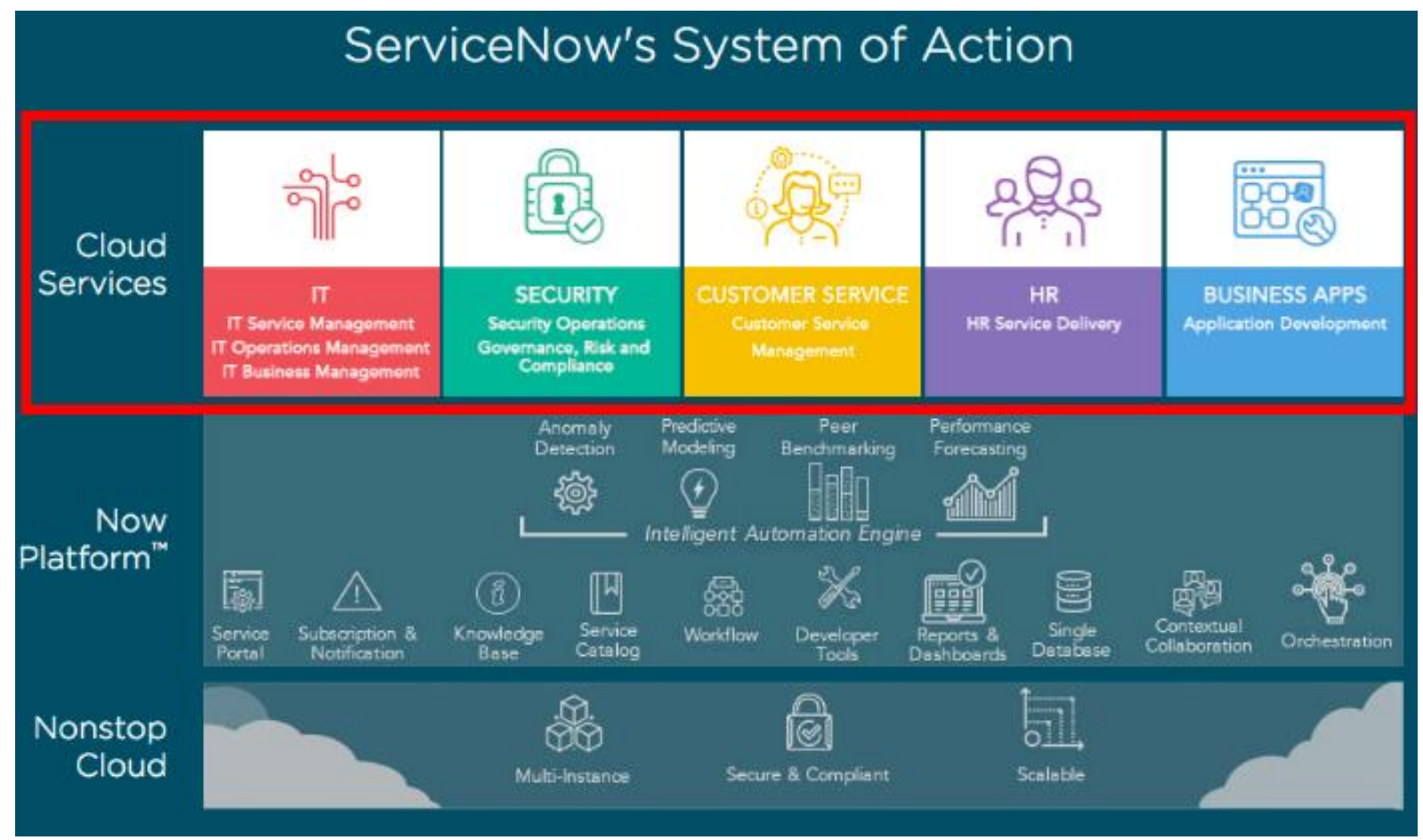

Fonte: SERVICENOW (2018)

Quadro 1 - Informações utilizadas para registro de ocorrências na plataforma ServiceNow.

\begin{tabular}{|c|c|c|c|}
\hline Caso de Uso & Ator & Objetivo & Canal \\
\hline Comunicar ocorrência & Cidadão & $\begin{array}{c}\text { Reportar uma ocorrência de } \\
\text { segurança pública }\end{array}$ & Aplicativo \\
\hline Rotear ocorrência & $\begin{array}{c}\text { Analista da Central } \\
\text { de Atendimento }\end{array}$ & $\begin{array}{c}\text { Direcionar a ocorrência de } \\
\text { acordo com a localidade e } \\
\text { habilidade policial necessária }\end{array}$ & Portal \\
\hline Receber ocorrência & Agente policial & $\begin{array}{c}\text { Ser informado da ocorrência } \\
\text { atribuída }\end{array}$ & $\begin{array}{c}\text { Aplicativo ou } \\
\text { Portal }\end{array}$ \\
\hline Tratar ocorrência & Agente policial & Tratar a ocorrência & - \\
\hline $\begin{array}{c}\text { Concluir ou reabrir } \\
\text { ocorrência }\end{array}$ & Cidadão & Concluir ou reabrir ocorrência & Aplicativo \\
\hline
\end{tabular}

Fonte: Os autores

O fluxograma de registro e resolução de ocorrências está ilustrado por meio da Figura 2, demonstrando possíveis atores e ações. Por exemplo, inicialmente o cidadão reporta uma ocorrência, através do aplicativo da Plataforma ServiceNow, que é exibida no painel de ocorrências da Central de Atendimento, que a avalia e a direciona para o Batalhão de Polícia, que a roteia para o agente policial. O agente, então, recebe uma notificação no aplicativo de celular, atende a chamada e a atualiza na plataforma. Assim, o cidadão é informado sobre a situação da ocorrência podendo ou concluir ou reabrir o registro da mesma.

As informações que seguem o fluxograma da Figura 2 podem ser visualizadas em ambiente de aplicativo de celular, como demonstrado na Figura 3, que exibe a versão observada pelo cidadão que registra a ocorrência, sendo, da esquerda para direita, o ícone do aplicativo ServiceNow na tela de seleção, tela de entrada do aplicativo, tela inicial, histórico de ocorrências reportadas, formulário de registro de nova ocorrência e notificação sobre atualização da ocorrência. 
Figura 2 - Fluxograma do processo de ocorrência de segurança pública.

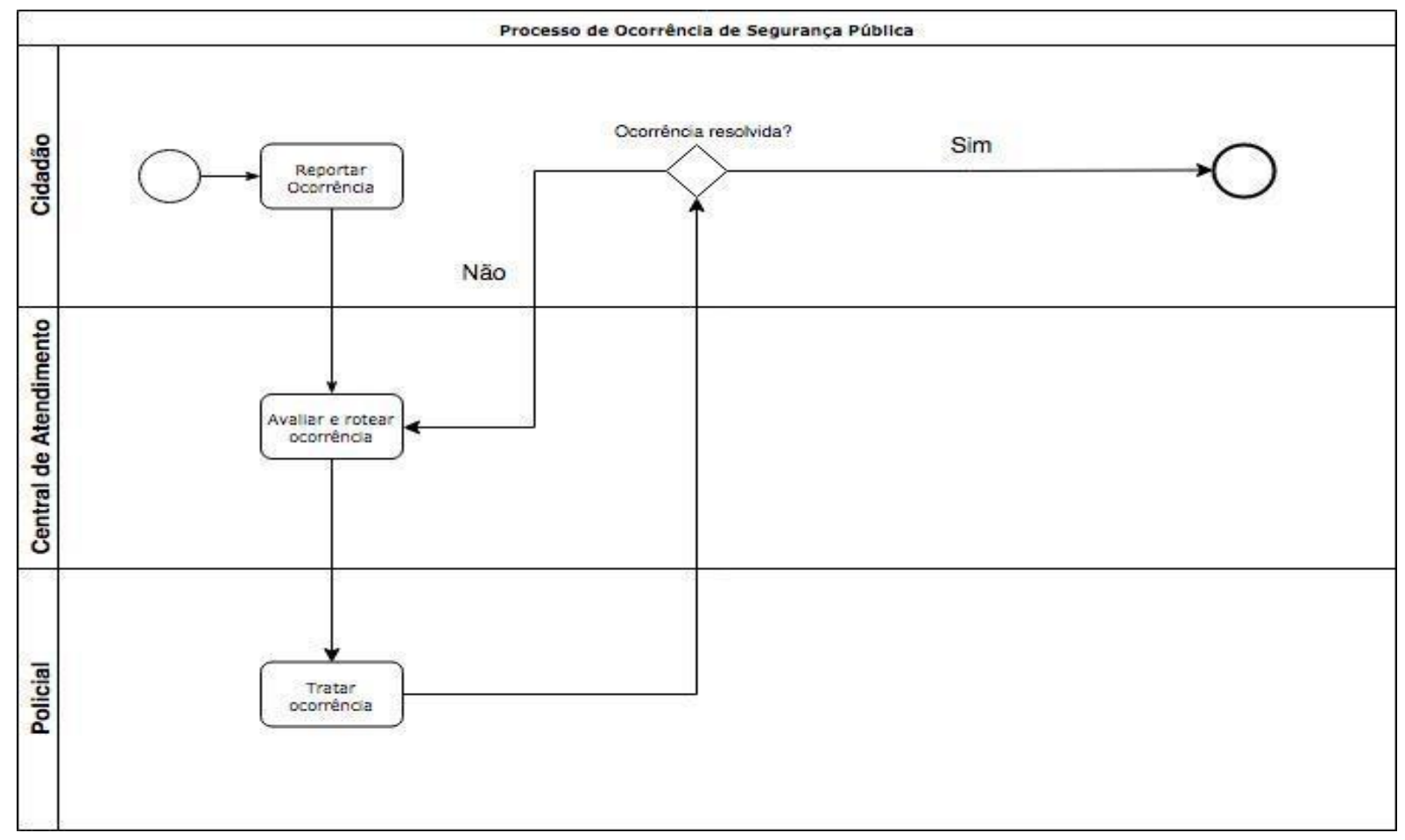

Fonte: Os autores

Figura 3 - Telas do Aplicativo na Visão do Cidadão.

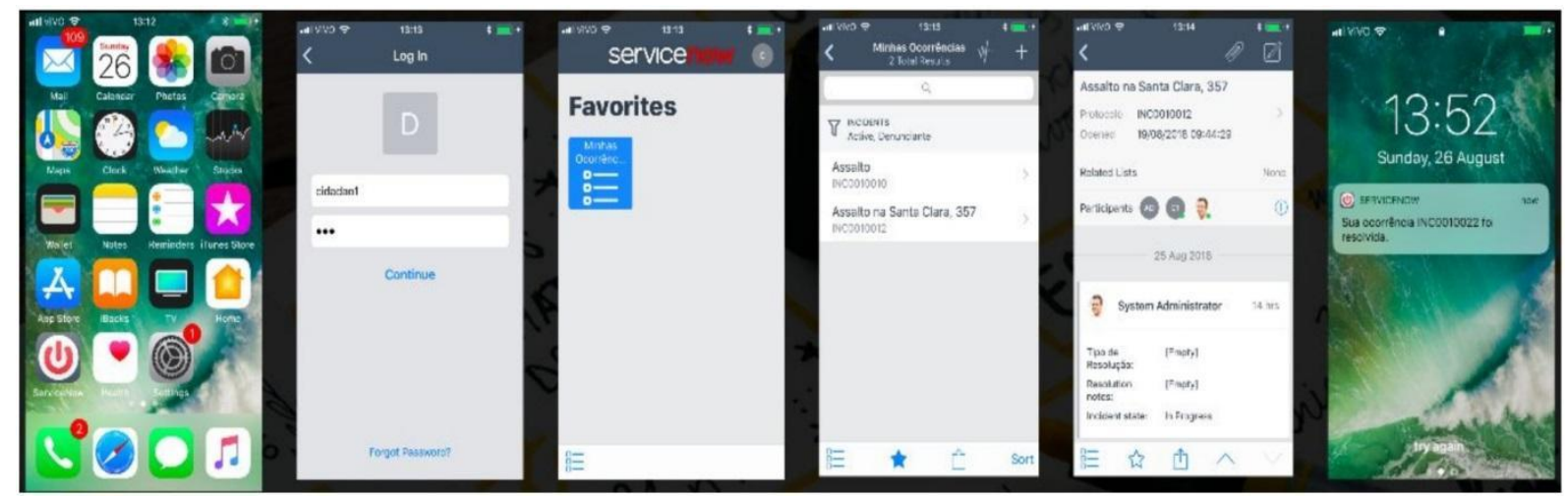

Fonte: Os autores

O recebimento de novas ocorrências e o controle das ocorrências em análise ou concluídas, realizado pela Central de Atendimento, pode ser facilmente administrado por meio de uma interface interativa, onde são exibidas: a lista de ocorrências e um histórico das ocorrências por categoria, que tem como objetivo auxiliar na tomada de decisões para minimização dos índices de criminalidade.

Após recebimento da ocorrência, o analista da Central de Atendimento deve encaminhar a informação para o Batalhão Policial correspondente. Para tal, tem-se um formulário onde são preenchidas determinadas informações, tais como: Canal, Categoria, Batalhão Policial, etc.

O agente policial deve, fundamentalmente, utilizar a plataforma por meio do aplicativo de celular. A Figura 4 exibe a interface deste aplicativo, sendo, da esquerda para direita, a tela com recebimento de notificação, a tela inicial da plataforma, a lista de ocorrências atribuídas ao agente policial e o formulário de atualização da ocorrência. 
Figura 4 - Interface do aplicativo para o agente policial.

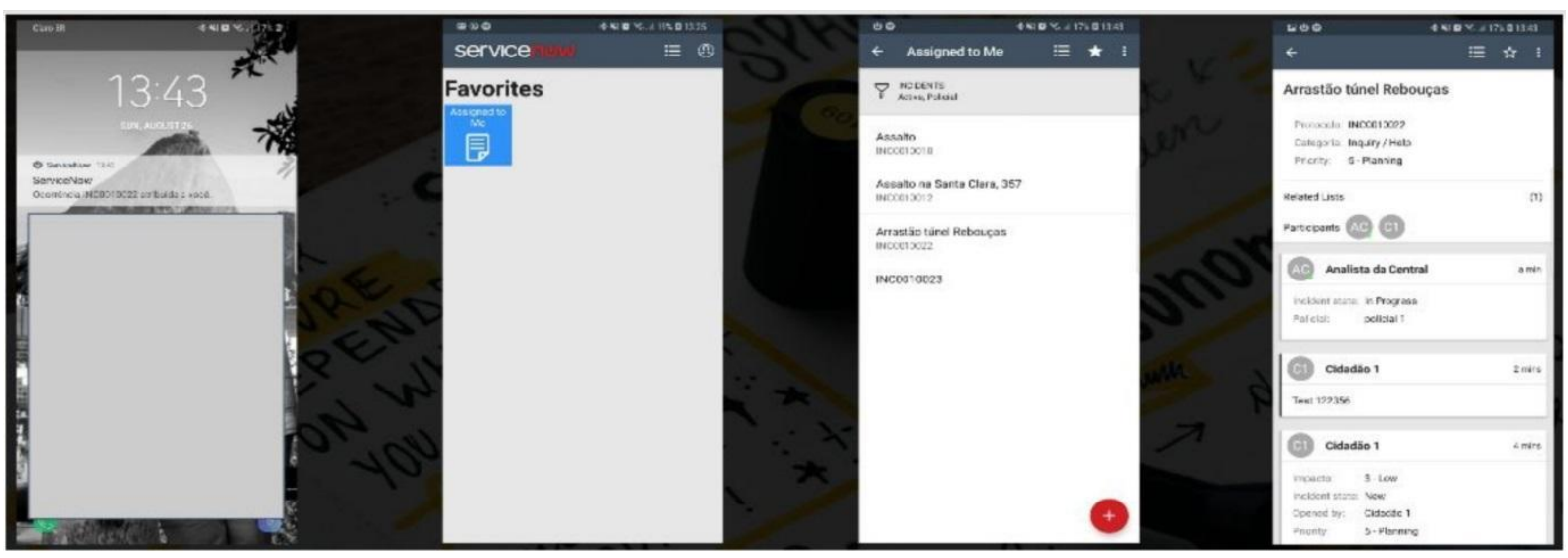

Fonte: Os autores

Vale ressaltar que essa proposta seria uma opção adicional aos meios já existentes no tratamento de ocorrências, como o 190. A adoção de um sistema/software privado pode ser dificultada pela questão de custos, mas nada impede que se faça uma adaptação para plataformas de acesso livre. Independente da forma de acesso, acredita-se que os impactos na gestão pública, em particular na segurança, seriam positivos. Notadamente, para utilização em massa de tal ferramenta seriam recomendados mais testes.

\section{CONSIDERAÇÕES FINAIS}

A plataforma ServiceNow pode ser uma potente ferramenta para o registro, monitoramento, direcionamento e tratamento de ocorrências, sendo acessível tanto por meio de aplicativos de celular quanto em interfaces de computador. A plataforma possibilita um fluxograma prático, rápido e eficaz desde o registro da ocorrência pelo cidadão comum até a resolução pelo agente policial designado, incluindo o registro no Batalhão Policial correspondente à ocorrência, ainda notificando ao cidadão a condição da mesma e a decisão sobre a necessidade ou não de reabertura do registro. A plataforma apresenta recursos que podem ser customizados, facilitando o desenvolvimento de novas abordagens.

Os custos da aquisição e da manutenção das licenças da plataforma ServiceNow podem inviabilizar a aplicação prática da mesma, mas a proposta apresentada pode ser adaptada em outras plataformas de acesso livre. A elaboração de um projeto piloto em uma pequena porção urbana é vital para verificar a real capacidade da plataforma em ambiente real, possibilitando o desenvolvimento de novas ferramentas e técnicas.

Outras funcionalidades da plataforma poderiam ser exploradas, tais como padronização do atendimento de ocorrências (Base de conhecimento), envio de notificações para outros fins e também por e-mail e mensagem de celular (SMS), elaboração de um mapa geográfico de ocorrências, direcionamento automático para batalhões policiais em função da experiência adquirida (Inteligência Artificial), uso de métricas e indicadores para os setores de inteligência da polícia e para acesso público, bem como pesquisa de satisfação do atendimento das ocorrências.

\section{REFERÊNCIAS}

ANDRADE, V. R. P. A mudança do paradigma repressivo em segurança pública: reflexões criminológicas críticas em torno da proposta da la Conferência Nacional Brasileira de Segurança pública. Sequência (Florianópolis), No.67, dez. 2013. DOI 10.5007/2177$7055.2013 v 34 n 67 p 335$. 
AZEVEDO, M. A. de. Concepções sobre criminalidade e modelos de policiamento. Psicologia: Ciência e Profissão, Vol.23, No.3, 2003, pp. 18-25. DOI 10.1590/S141498932003000300004.

AZEVEDO, R. G. Prevenção integrada: novas perspectivas para as políticas de segurança no Brasil. Revista Katálysis, Florianópolis, Vol.9, No.1, jan./jun. 2006. DOI 10.1590/S141449802006000100004

CARVALHO, V. A. D.; SILVA, M. D. R. Política de segurança pública no Brasil: avanços, limites e desafios. Revista Katálysis, Florianópolis, Vol.14, No.1, jan./jun. 2011 . DOI 10.1590/S141449802011000100007.

FURTADO, V. Tecnologia e gestão da informação na segurança pública. Ed. Garamond, Rio de Janeiro, Brasil, 2002.

GARTNER. Magic quadrant for enterprise high-productivity application platform as a service. Gartner, 26 abr. 2018. Disponível em: <https://www.gartner.com/doc/reprints?id=1 4UCCMEP \& $c t=180329 \&$ st=sb>. Acesso em: 03 set. 2018.

IPEA. Instituto de Pesquisa Econômica Aplicada. Relatório Final da $1^{a}$ Conferência Nacional de Segurança Pública. Ministério da Justiça, Brasília - DF, 27 a 30 de agosto de 2009. Disponível em: <http://www.ipea.gov.br/participacao/images/pdfs/conferencias/ Seguranca_Publica/relatorio_final_1_conferencia_seguranca_publica.pdf>. Acesso em: 03 set. 2018

LIMA, R. S. D.; BUENO, S.; MINGARDI, G. Estado, polícias e segurança pública no Brasil. Revista Direito GV, São Paulo, Vol.12, No. 1, jan./abr. 2016. DOI 10.1590/2317-6172201603.

MÁXIMO, A. A. A importância do mapeamento da criminalidade utilizando-se tecnologia de sistema de informação geográfica para auxiliar a segurança pública no combate à violência. Universidade Federal de Santa Catarina, Florianópolis, 2004.

PACHECO, B. C. Tecnologias de Informação e Comunicação para a Democracia: uma Análise Comparativa das Estratégias do Poder Executivo dos Municípios do Rio de Janeiro e de São Paulo (2013-2016). Dissertação de Mestrado. Universidade Federal do Rio de Janeiro, Instituto de Economia, Programa de Pós-Graduação em Políticas Públicas, Estratégias e Desenvolvimento, 2018. $131 \mathrm{p}$.

REULAND, M. M. Information management and crime analysis: practitioners's recipes for success. Police Executive Research Forum, Whashington, D.C., EUA, 1997.

SERVICENOW. ServiceNow Trust and Compliance Center: Operations and Availability. ServiceNow, 2018. Disponível em: <https://www.servicenow.com/now-platform.html>. Acesso em: 03 set. 2018. 